\section{Imagery-induced McCollough effects: Real or imagined?}

\section{SETH KUNEN and JAMES G. MAY \\ University of New Orleans, New Orleans, Louisiana 70122}

Recently, this journal published two studies, one by Broerse and Crassini (1980) and one by us (Kunen \& May, 1980), that report contradictory findings on whether visual imagery can be used to induce contingent color aftereffects (CAEs). We found, using a color-cancellation procedure, that CAEs could be produced from adaptation to visual imagery. These findings replicate and extend earlier findings of imagery-induced CAEs reported by Finke and Schmidt (1977, 1978). In contrast, Broerse and Crassini (1980), using a forced-choice verbal report paradigm, report that no CAEs could be obtained from adaptation to visual imagery and that interocular transfer of CAEs did not occur. ${ }^{1}$

Finke (1981), in commenting on these articles, suggests that the failure of Broerse and Crassini to find CAEs after adaptation to visual imagery might have reflected their use of a forced-choice method of measuring CAEs. We also suspect that their failure to find imagery-induced CAEs might have resulted from the particular method they employed. Broerse and Crassini told subjects that subsequent to adaptation they would view a test slide and would have to judge whether the test slide contained color and, if color was present, to rate the degree to which the color was present. Such a procedure may have enabled subjects to easily perceive a relationship between the study and test phases and to try to secondguess the experimenters. For example, after imagining patterns, the subjects may have figured out that there was no objectively present color in the test slides and may have felt that they were being tested for the extent to which they could be coaxed into believing that colors were present. In our colorcancellation procedure, it was virtually impossible for naive subjects to guess what our experimental hypotheses were or to see any relationship between the color-cancellation adjustments and the patterns in their imagery. Also, the color-cancellation procedure may measure CAEs more directly than verbal reports and thus may be a more sensitive method.

The results of our study indicated that visual imagery can be a source of visual stimulation that induces CAEs. Furthermore, the direction of the CAEs derived from imagery was opposite to the direction of CAEs resulting from adaptation to well-focused externally present stimuli. Since it is well established that, with adaptation to checkerboard patterns of $3.0 \mathrm{c} / \mathrm{d}$, CAEs are derived from adaptation to the funda- mental spatial frequencies, we interpreted our imageryinduced CAEs as being derived from the higher spatial frequencies (May, Agamy, \& Matteson, 1978). However, when subjects imaged blurred versions of the checkerboards, which makes the lower spatial frequencies noticeable, the direction of the imagery CAEs was reversed and indicated adaptation, as with externally induced CAEs to lower spatial frequencies.

Although our conclusion that subjects must be aware of specific spatial frequency information before they can incorporate such information into their imagery is somewhat speculative, we felt this explanation best fit the data. Finke (1981) has disagreed with our interpretation and has suggested instead that an information overload resulted in the emphasis of local edge information. This interpretation deserves serious consideration but does not seem consistent with several aspects of perceptual processing. First, although it is true that subjects are presented with hundreds of checks, this need not present a situation of overload (cf. Weisstein \& Harris, 1980). Since the checkerboard is extremely redundant, a subject need only visualize a small segment in order to represent all the information in the checkerboard. Second, with a high-spatial-frequency checkerboard (e.g., $5.0 \mathrm{c} / \mathrm{d}$ fundamental) displayed in a 10-15-deg field, it is apparent from careful inspection that only a handful of squares around the point of fixation (about $2 \mathrm{deg}$ ) are perceived as having well-defined edges. If one attends to more peripheral portions of the field, the fundamental Fourier components (which appear at 45-deg angles to the edges) are perceived. Thus, even with actual viewing, only a handful of squares provide edge information, but Fourier effects are obtained from adaptation experiments with such stimuli. It is unlikely that Finke's prediction concerning imagery with stimuli of reduced area will be upheld, and, even if it is, there are other interpretations available in addition to "limited capacity." May, Harter, and Siegfried (1981) have shown that Fourier effects are greater in magnitude when test stimuli are presented to the periphery rather than to the fovea. It may be that subjects who are given the task of imaging patterns choose to image the visual features that are normally abstracted by the fovea and must be specifically instructed to attend to more peripherally processed aspects before all the relevant features can be represented.

In conclusion, we would like to interject a word of caution, since at present, as far as we know, there have only been four published studies on imageryinduced McCollough effects (Broerse \& Crassini, 1980; Finke \& Schmidt, 1977, 1978; Kunen \& May, 1980). This area of research is highly intriguing, since 
it strives to combine cognition and vision in a unique way; as such, the CAEs being studied may be sensitive to a number of factors not systematically explored yet. For example, the role of how forcefully the imagery instructions are formulated and administered is a factor that has been unexplored and is likely to be of considerable importance in such experiments. We feel that these four studies raise many provocative questions, but, before any definitive answers are proposed, more research is needed.

\section{REFERENCE NOTE}

1. May, J. G., Kaufman, J., \& Kunen, S. Interocular transfer of the McCollough effect. Paper presented at the 21 st Annual Meeting of the Psychonomic Society, Phoenix, Arizona, November 1980.

\section{REFERENCES}

Broense, J., \& Crassini, B. The influence of imagery ability on color aftereffects produced by physically present and imagined induction stimuli. Perception \& Psychophysics, 1980, 28, 560-568.

Finke, R. A. Interpretations of imagery-induced McCollough effects. Perception \& Psychophysics, 1981, 30, 94-95.

Finke, R. A., \& SchмidT, M. J. Orientation-specific color after- effects following imagination. Journal of Experimental Psychology: Human Perception and Performance, 1977, 3, 599-606. Finke, R. A., \& Schmidt, M. J. The quantitative measure of pattern representation in images using orientation-specific color aftereffects. Perception \& Psychophysics, 1978, 23, 515-520.

KunEN, S., \& MAY, J. G. Spatial frequency content of visual imagery. Perception \& Psychophysics, 1980, 28, 555-559.

May, J. G., Agamy, G., \& Matteson, H. H. The range of spatial frequency-contingent color aftereffects. Vision Research, $1978,18,917-921$.

May, J. G., Harter, M. R., \& Siegfried, J. B. Electrophysiological and psychophysical measures of interocular suppression. Psychophysiology, 1981, 18, 26-31.

Weisstein, N., \& HARris, C. S. Masking and the unmasking of distributed representations in the visual system. In C. S. Harris (Ed.), Visual coding and adaptability. Hillsdale, N.J: Erlbaum, 1980.

\section{NOTE}

1. We have recently reported at the annual meeting of the Psychonomic Society that imagery-induced CAEs transfer interocularly under conditions that do not give rise to the transfer of CAEs proposed by external adaptation (May, Kaufman, \& Kunen, 1980, Note 1).

(Manuscript received May 18, 1981; accepted for publication June 17, 1981.) 